\title{
PROGRAMA DE REENTRENAMIENTO EN DIÁLISIS
} PERITONEAL AMBULATORIA

\author{
Lila Quintero*, María Mercedes Martínez**, María Rita Melo**, Zoila María Silva** Alexandra Chaparro.**
}

\section{Resumen}

Con el fin de evaluar la influencia de un programa de reentrenamiento de los conocimientos teoricoprácticos del recambio del líquido peritoneal en pacientes con diálisis peritoneal de la unidad renal del hospital San Carlos, se llevó a cabo el presente estudio en 35 pacientes, con edades de 18 a 76 años y con un tiempo en el programa de diálisis peritoneal ambulatoria continua (CAPD) mayor de dos meses.

Es un estudio descriptivo, cuantitativo, cuasiexperimental y longitudinal. Se aplicaron dos instrumentos, un cuestionario para evaluar los conocimientos teóricos y una lista de comprobación para evaluar la técnica del recambio peritoneal. Los instrumentos se aplicaron antes y después del programa de reentrenamiento.

Al aplicar los primeros se observó que el 55\% de los pacientes tienen buenos conocimientos teoricoprácticos del recambio peritoneal, un $26 \%$ demostró una práctica regular y solo un 3\% deficiencias en la teoría; con base en estos resultados se vió la necesidad de reforzar estos conocimientos a través de un programa, reentrenando a los 35 pacientes.

Después del programa de reentrenamiento se observó el cambio en los conocimientos teoricoprácticos, desapareciendo el rango deficiente en la evaluación de los conocimientos teóricos y disminuyendo el regular en la práctica en un $50 \%$.

Para el análisis de los datos estadísticos se emplearon los programas SPSS y Excel, utilizando tablas y gráficas de cajas. Se concluyó que el programa de reentrenamiento influyó en forma positiva en los conocimientos teoricoprácticos del recambio peritoneal ya que los pacientes modificaron sus comportamientos mejorando las categorías de bueno y excelente. Palabras clave: diálisis peritoneal ambulatoria continua (CAPD), programa de reentrenamiento, recambio del líquido peritoneal, unidad renal.

Abreviaturas: CAPD, diálisis peritoneal ambulatoria continua; APD, diálisis peritoneal automatizafa.

\section{Introducción}

La unidad renal del Hospital San Carlos cuenta con los programa de hemodiálisis y diálisis peritoneal para el tratamiento de la insuficiencia renal aguda y/o crónica; la diálisis peritoneal presenta dos modalidades de tratamiento: CAPD (diálisis peritoneal ambulatoria continua) y APD (diálisis peritoneal automatizada). Ambos son de fácil acceso para el paciente, ya que se realiza en el hogar de forma manual (CAPD) ó automatizada (APD).

\footnotetext{
Fecha recibido: enero 18 de 2006-Fecha aceptado: junio I5 de 2006.
}

* Enfermera, profesora asociada, facultad de enfermería de la Fundación Universitaria de Ciencias de la Salud, Bogotá. DC

** Enfermera, estudiante de la especialización en nefrología y urología, facultad de enfermería de la Fundación Universitaria de Ciencias de la Salud, Bogotá. DC.

Investigación realizada en el Hospital San Carlos de Bogotá, D.C. durante el segundo semestre de 2004.
La mayoría están en CAPD y requieren un entrenamiento teoricopráctico, además de adecuadas condiciones socio culturales y de vivienda para llevar a cabo el procedimiento de los recambios del líquido peritoneal en el hogar. Es una terapia que exige gran compromiso y responsabilidad, en especial con su autocuidado.

Una de las principales complicaciones que pone en peligro la vida del paciente con este tratamiento es la peritonitis, que constituye la primera causa de morbimortalidad en el programa de diálisis peritoneal.

Observando la incidencia de peritonitis del 56\% en el período de enero a septiembre del 2004 en 76 pacientes del programa de diálisis peritoneal, el grupo investigador planteó como objetivo evaluar los conocimientos teoricoprácticos del "recambio del líquido peritoneal", con el propósito de verificar si hay deficiencia en la técnica y conocimientos del procedimiento, ya que la peritonitis se produce a 
menudo, debido a una técnica inapropiada durante la conexión y desconexión de ultrabag, esto facilita a las bacterias la entrada a la cavidad peritoneal a través de la luz del catéter. El germen que con mayor frecuencia se ha aislado en los pacientes con peritonitis es el staphylococcus epidermidis con una incidencia del 30 al $45 \%$. Para verificar los conocimientos teoricoprácticos se utilizaron dos mediciones, para los teóricos se aplicó una encuesta y para la técnica del recambio peritoneal una lista de chequeo; de acuerdo con los resultados de la primera medición se diseñó y se aplicó un programa de reentrenamiento; la segunda medición se aplicó después de la intervención del programa de reentrenamiento. La muestra de pacientes fue de 35 .

Es un trabajo de investigación descriptivo, cuantitativo de intervención tipo cuasiexperimental y longitudinal.

Es el primer trabajo de la unidad renal del Hospital San Carlos, que evalúa la técnica del recambio peritoneal, por lo tanto es novedoso y de gran aporte para el profesional de enfermería, que es el responsable directo del entrenamiento de los pacientes, además de determinar los correctivos necesarios individuales con el fin de prevenir complicaciones, en especial la peritonitis.

\section{Métodos}

Al iniciar la investigación se contaba con una población total de 76 pacientes, pero al aplicar los criterios de exclusión e inclusión disminuyeron a 52. Al aplicar los instrumentos de medición se observó que algunos habían sido trasladados a otras unidades de diálisis por razones administrativas de las EPS correspondientes, quedando un total de 38 pacientes, de los cuales no se pudieron entrevistar tres porque no cumplieron con las citas acordadas, quedando en total 35 pacientes; esta muestra fue no probabilística por conveniencia.

Como criterios de inclusión se consideraron los pacientes mayores de 18 años y con un período de permanencia en CAPD mayor de dos meses. Los criterios de exclusión fueron los pacientes en programa APD y los recién entrenados en CAPD. Se aplicaron dos instrumentos, un cuestionario para los conocimientos teóricos y una lista de comprobación de los prácticos; fueron diseñados e implementados por las investigadoras y validados por personas expertas en diálisis peritoneal. La prueba piloto se aplicó en cinco pacientes de la unidad renal que no participaron de la investigación.

El cuestionario consta de once preguntas de selección múltiple, con una respuesta verdadera. Los criterios para evaluar fueron, excelente once puntos, bueno de diez a ocho puntos, regular de siete a seis puntos y deficiente menor de cinco puntos

Los conocimientos prácticos se evaluaron por medio de una lista de 40 ítems. Cada uno fue analizado de acuerdo con el orden de importancia y se ponderó de la siguiente forma: a los 30 pasos indispensables para la realización del recambio peritoneal que tienen mayor riesgo para la peritonitis se les dio un valor de 1.0. Los diez procedimientos restantes tuvieron un valor de 0.5 puntos.

El valor total de la encuesta fue de 35.5 puntos y se evaluó así: excelente 35.5 puntos, bueno $35-28$, regular $27-21$ y deficiente menor de 20 .

La ponderación para evaluar la lista fue analizada por las enfermeras del programa de diálisis peritoneal del Hospital San Carlos. Para evaluar la práctica, el paciente realizó el recambio peritoneal con el material de simulación del programa; una de las investigadoras observó el procedimiento y otra lo registraba en la lista de comprobación.

Una vez aplicada la primera encuesta y la lista de comprobación se analizaron los resultados, se identificaron las falencias y de acuerdo con estas, se diseñó el programa de reentrenamiento. Después de un mes de implementado este programa, se aplicaron por segunda vez los mismos instrumentos, la encuesta y la lista de chequeo, para determinar la influencia del programa.

Los primeros instrumentos se aplicaron en la primera semana de noviembre de 2004; el programa de reen- 
trenamiento se diseñó y se implementó durante las primeras dos semanas del mes de diciembre de este mismo año y los segundos instrumentos se aplicaron en las primeras dos semanas de enero de 2005.

\section{Resultados}

Los resultados estadísticos fueron obtenidos por medio del programa SPSS software para estadística social y Excel. Para la presentación de los resultados se utilizaron tablas y gráficas de cajas.

Características socio-demográficas: dentro de las principales características socio demográficas, se encontró que el $40 \%$ de la muestra estaba conformada por mujeres con una edad mediana de 38.5 años, con una mínima de 21 y máxima de 76 , la edad mediana de los hombres fue de 59 años variando entre 19 y 70 (Tabla 1).

Referente al estado civil, eran casados el $46 \%$, solteros $26 \%$ y viudos el $6 \%$ (dos personas) (Tabla 2 ).

En la convivencia, cohabitan con su cónyuge e hijos el $26 \%$, seguido del $23 \%$ de aquel que vive solo con su cónyuge y en una menor proporción (14\%) con familiares, hijos o padres (Tabla 3).

En cuanto al nivel educativo de los pacientes, se encontró que el $29 \%$ tienen primaria completa,

\begin{tabular}{|c|c|c|c|c|}
\hline \multicolumn{5}{|c|}{ Tabla I. Distribución por sexo $y$ edad. } \\
\hline \multirow{2}{*}{ Sexo } & Numero de pacientes & Porcentaje & Promedio & Desviación \\
\hline F & 14 & $40 \%$ & 41,1 & 17,2 \\
\hline M & 21 & $60 \%$ & 52,9 & 14,8 \\
\hline Total general & 35 & $100 \%$ & 48,1 & 16,6 \\
\hline
\end{tabular}

\begin{tabular}{|c|c|c|}
\hline \multicolumn{3}{|c|}{ Tabla 2. Distribución de la muestra según su estado civil. } \\
\hline Estado civil & Número de pacientes & porcentaje \\
\hline Casado & 16 & $46 \%$ \\
\hline Soltero & 9 & $26 \%$ \\
\hline Unión libre & 5 & $14 \%$ \\
\hline Separado & 3 & $9 \%$ \\
\hline Viudo & 2 & $5 \%$ \\
\hline Total general & 35 & $100 \%$ \\
\hline
\end{tabular}

Tabla 3. Convivencia de los pacientes evaluados

\begin{tabular}{|c|c|c|}
\hline \multicolumn{2}{|c|}{ Tabla 3. Convivencia de los pacientes evaluados. } \\
\hline Convivencia & Número de pacientes & Porcentaje \\
\hline Cónyuge-hijos & 9 & $26 \%$ \\
\hline Cónyuge & 8 & $23 \%$ \\
\hline Familiares & 5 & $14 \%$ \\
\hline Hijos & 5 & $14 \%$ \\
\hline Padres & 5 & $14 \%$ \\
\hline Solo & 2 & $6 \%$ \\
\hline Padres y familiares & 1 & $3 \%$ \\
\hline Total general & 35 & $100 \%$ \\
\hline
\end{tabular}


seguido del $17 \%$ con primaria incompleta, $14 \%$ son analfabetas, $14 \%$ con estudios secundarios incompletos y solo el $6 \%$ tienen estudios universitarios (dos personas)(Tabla 4).

En cuanto al tiempo de permanencia en el programa de CAPD se halló que la mayoría llevaban entre dos

\begin{tabular}{|l|c|c|}
\hline \multicolumn{3}{|c|}{ Tabla 4. Nivel escolar de los pacientes. } \\
\hline Primaria completa & $\begin{array}{c}\text { Número de } \\
\text { pacientes }\end{array}$ & Porcentaje \\
\hline Primaria incompleta & 10 & $29 \%$ \\
\hline Analfabeta & 6 & $17 \%$ \\
\hline $\begin{array}{l}\text { Secundaria } \\
\text { incompleta }\end{array}$ & 5 & $14 \%$ \\
\hline Secundaria completa & 4 & $14 \%$ \\
\hline Técnico & 3 & $9 \%$ \\
\hline Universitario & 2 & $6 \%$ \\
\hline Total general & 35 & $100 \%$ \\
\hline
\end{tabular}

\begin{tabular}{|l|c|c|}
\hline \multicolumn{3}{|c|}{ Tabla 5. Tiempo de permanencia dentro del programa } \\
Te CAPD. \\
& $\begin{array}{c}\text { No. de } \\
\text { pacientes }\end{array}$ & Porcentaje \\
\hline 2-6 meses & 13 & $37 \%$ \\
\hline 6 meses- I año & 13 & $37 \%$ \\
\hline 1-2 años & 3 & $9 \%$ \\
\hline 2-3 años & 2 & $6 \%$ \\
\hline Más de tres años & 4 & $11 \%$ \\
\hline Total general & 35 & $100 \%$ \\
\hline
\end{tabular}

\begin{tabular}{|l|c|c|}
\hline $\begin{array}{c}\text { Tabla 6. Tablas de frecuencia. Conocimientos teóricos del } \\
\text { recambio peritoneal antes del reentrenamiento. }\end{array}$ \\
\hline $\begin{array}{c}\text { Nonel de } \\
\text { teórico }\end{array}$ & $\begin{array}{c}\text { No. de } \\
\text { pacientes }\end{array}$ & $\begin{array}{c}\text { Distribución } \\
\text { porcentual }\end{array}$ \\
\hline Excelente & 14 & $40 \%$ \\
\hline Bueno & 15 & $43 \%$ \\
\hline Regular & 5 & $14 \%$ \\
\hline Deficiente & 1 & $3 \%$ \\
\hline
\end{tabular}

meses y un año, (74\%), el 9\% de 1 a 2 años, el 6\% de 2 a 3 años y el 11\% más de 3 años (Tabla 5).

Preentrenamiento del recambio del líquido peritoneal: los conocimientos teóricos sobre el recambio peritoneal, el $40 \%$ fueron excelentes, el $40 \% 43 \%$ buenos, $14 \%$ regulares y el $3 \%$ deficientes. (Tabla 6).

Con relación a la lista de comprobación del procedimiento, el $11 \%$ practicaron el recambio peritoneal en forma excelente, el $63 \%$ realizaron una buena práctica y el $26 \%$ una regular porque omitieron varios pasos (Tabla 7).

\begin{tabular}{|l|c|c|}
\hline $\begin{array}{c}\text { Tabla 7. Nivel de conocimiento práctico del recambio } \\
\text { peritoneal antes del reentrenamiento. }\end{array}$ \\
\hline $\begin{array}{c}\text { Nivel de } \\
\text { práctico }\end{array}$ & $\begin{array}{c}\text { No. de } \\
\text { pacientes }\end{array}$ & $\begin{array}{c}\text { Distribución } \\
\text { porcentual }\end{array}$ \\
\hline Excelente & 4 & $11 \%$ \\
\hline Bueno & 22 & $63 \%$ \\
\hline Regular & 9 & $26 \%$ \\
\hline
\end{tabular}

Analizando cada pregunta del cuestionario y los ítems de la lista de chequeo los resultados fueron:

Cuestionario de conocimientos teóricos: el 49\% olvidó la función del catéter de la diálisis peritoneal, el cual se evaluó a través de la pregunta siete. El $31 \%$ falla en la identificación de los elementos para realizar el recambio del líquido peritoneal, (pregunta cuatro). El 20\% no observa la fecha de vencimiento, la integridad de los empaques y de los elementos a utilizar antes del procedimiento peritoneal, (pregunta diez).

Lista de comprobación: el 49\% no efectúa un lavado de manos al finalizar el procedimiento del recambio peritoneal. El 46\% incumple con la verificación del ultrabag: fugas, líneas, anillo protector, solución y bolsa de drenaje. El 34\% ejecuta la conexión al ultrabag sin cumplir con la colocación de la mano de acuerdo con la técnica de cigarrillo recomendada en el protocolo. El 20\% mantiene la punta de la línea de transferencia hacia arriba en el momento de colocar el nuevo minicap. 
Resultado del programa de reentrenamiento: teniendo en cuenta las deficiencias en los conocimientos teóricos y prácticos del recambio peritoneal se reforzó la importancia de cumplir con todos los aspectos, ya que su omisión conlleva complicaciones que repercuten en el bienestar y en la vida del paciente, lo cual justificó el programa de reentrenamiento.

Al mes siguiente de implementarse el programa se aplicaron los mismos instrumentos que se manejaron en la primera medición, es decir, la encuesta y lista de chequeo.

Recordemos los argumentos de Cruz Cosme en el libro de "Diálisis Peritoneal" en el capitulo de educación de adultos, cuando se refiere al entrenamiento de los pacientes anota que se suele realizar en condiciones inadecuadas físicas y psicológicas, y esta situación conlleva a la memorización y no a la comprensión de la importancia del entrenamiento, implicando el olvido rápido de lo enseñado. Además las investigaciones pedagógicas indican que la curva de olvido de lo memorizado es: a los siete días se olvida un tercio de lo aprendido, a los veintiún días las dos terceras partes y a los treinta días se olvida prácticamente todo, razón por la cual se aplicó el segundo instrumento al mes de aplicar el programa de reentrenamiento.

Se encontró que el 57\% de los pacientes presentaron un conocimiento teórico excelente, el 31\% bueno y solo el $11 \%$ regular (Tabla 8). El 40\% realizaron una práctica excelente, el $49 \%$ demostraron una buena y el $11 \%$ una regular (Tabla 9).Los resultados anteriores muestran mejoría, desapareciendo el comportamiento deficiente en los conocimientos teóricos y disminuyendo el rango regular en la práctica en un $50 \%$. Los porcentajes de excelencia aumentaron en forma significativa.

\begin{tabular}{|c|c|c|}
\hline \multicolumn{2}{|c|}{$\begin{array}{c}\text { Tabla 8. Conocimientos teóricos del recambio peritoneal } \\
\text { después del reentrenamiento. }\end{array}$} \\
\hline $\begin{array}{c}\text { Nivel de } \\
\text { conocimiento } \\
\text { teórico }\end{array}$ & $\begin{array}{c}\text { Número de } \\
\text { pacientes }\end{array}$ & $\begin{array}{c}\text { Distribución } \\
\text { porcentual }\end{array}$ \\
\hline Excelente & 20 & $57.14 \%$ \\
\hline Bueno & 11 & $31.43 \%$ \\
\hline Regular & 4 & $11.43 \%$ \\
\hline
\end{tabular}

\begin{tabular}{|c|c|c|}
\hline $\begin{array}{c}\text { Tabla 9. Nivel de conocimiento práctico del recambio } \\
\text { peritoneal después del reentrenamiento. }\end{array}$ \\
\hline $\begin{array}{c}\text { Nivel de } \\
\text { conocimiento } \\
\text { práctico }\end{array}$ & $\begin{array}{c}\text { Número de } \\
\text { pacientes }\end{array}$ & $\begin{array}{c}\text { Distribución } \\
\text { porcentual }\end{array}$ \\
\hline Excelente & 14 & $40 \%$ \\
\hline Bueno & 17 & $49 \%$ \\
\hline Regular & 4 & $11 \%$ \\
\hline
\end{tabular}

Análisis comparativo antes y después del programa de reentrenamiento: para el análisis comparativo de los comportamientos excelente, bueno y regular se calificó de cinco (5.0) a cero (0.0) teniendo en cuenta los puntajes obtenidos, con tablas de frecuencia y gráficos de cajas.

En cuanto a los conocimientos teóricos, se encontró que el puntaje mediano mejoró pasando de 4.5 a 5.0; caso similar ocurrió con la puntuación mínima obtenida ya que esta fue de 1.4 antes del reentrenamiento y luego subió a 3.2 (Tabla 10). De la misma manera se notó que el rango de variación en la segunda fase fue menor (1.8), lo cual significa que los pacientes en dicha etapa tuvieron un nivel de conocimiento más homogéneo que en la primera, afirmación que se comprueba también al realizar un gráfico de cajas (Gráfico 1). Además, en dicho gráfico se detectaron en la segunda etapa dos pacientes que tuvieron un comportamiento atípico a los restantes, dado que su nivel de conocimiento osciló entre 3.2 a 3.5, obteniendo la concentración de puntajes entre 4.5 y 5 puntos.

En el análisis de cada uno de los diferentes ítems del cuestionario de conocimiento se identificó que la deficiencia en los puntos "función del catéter en la diálisis peritoneal" y "los elementos para realizar el recambio del liquido peritoneal" disminuyó en

\begin{tabular}{|c|c|c|c|c|}
\hline \multicolumn{5}{|c|}{$\begin{array}{c}\text { Tabla 10.Resumen de estadísticas descriptivas del puntaje de } \\
\text { conocimiento y práctica en } 35 \text { pacientes del Hospital San carlos }\end{array}$} \\
\hline Instrumento & Etapa & Mediana & Mínimo & Máximo \\
\hline \multirow{2}{*}{ Conocimiento } & Pre & 4.5 & 1.4 & 5 \\
\cline { 2 - 5 } & Post & 5.0 & 3.2 & 5 \\
\hline & Pre & 4.6 & 3.4 & 5 \\
\hline Práctica & Post & 4.9 & 3.5 & 5 \\
\hline
\end{tabular}




\section{CALIFICACIÓN DEL CUESTIONARIO DE CONOCIMIENTOS}

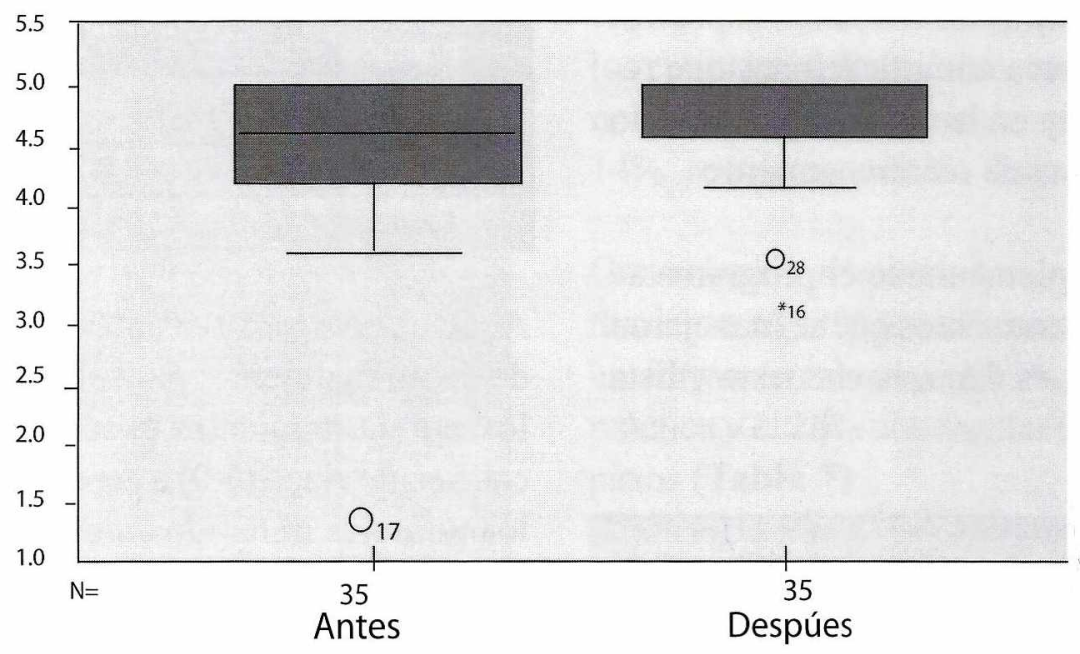

Gráfico I. Comparación de conocimientos teóricos del recambio peritoneal antes y después del programa de reentrenamiento.

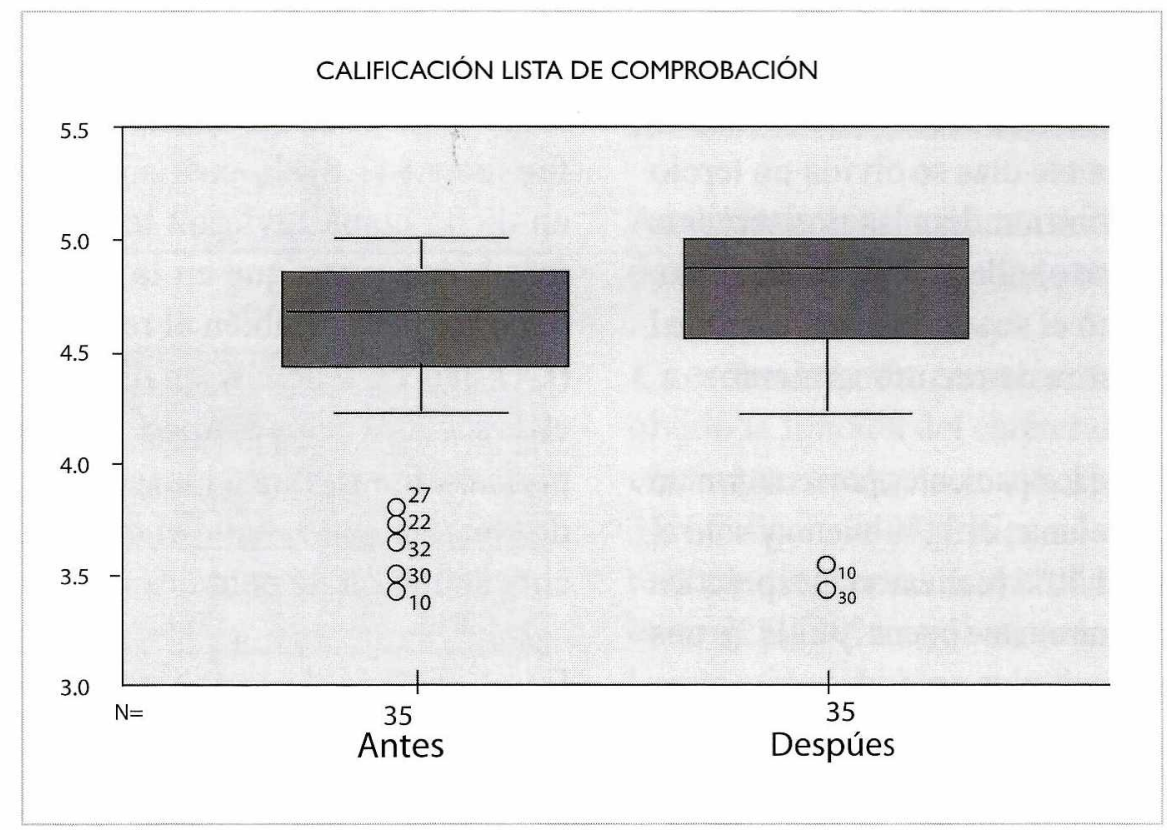

Gráfico 2. Comparación de conocimientos prácticos antes y después del programa de reentrenamiento.

forma evidente del $31 \%$ antes del entrenamiento al $18 \%$ después y del $20 \%$ del pre al $11 \%$ en el post. De igual manera el $20 \%$ de los pacientes no conocen ni antes ni después del reentrenamiento, los aspectos de la observación del dianeal (concentración indicada, color y verificación del empaque), es decir, hay que reforzar este aspecto.
Al comparar los conocimientos prácticos medidos con la lista de comprobación se observó que el puntaje mediano mejoró en 0.3 puntos, es decir pasó de 4.6 a 4.9 después del reentrenamiento (Tabla 10). También la variación se modificó ya que en la segunda fase fue menor, en otras palabras, el nivel de conocimiento práctico fue más homogéneo que 
en la primera medición. En el gráfico 2 se hallaron cinco pacientes en la primera y dos en la segunda etapa que tuvieron un comportamiento atípico. Vale la pena resaltar que los dos pacientes, identificados en la segunda etapa fueron los que presentaron el puntaje más bajo de conocimientos en la primera.

Al analizar cada uno de los ítems de la lista de comprobación en lo relacionado con "reúne los elementos, verifica empaques y fechas de vencimiento" ( $29 \%$ antes y $20 \%$ después), los pacientes mejoraron en relación con la primera medición. También se encontró que el 17\% no tiene la costumbre de limpiar dos veces la mesa con solución desinfectante ni divide la superficie en área estéril y limpia; al igual, el $20 \%$ de los pacientes no verifica las características ni mide el líquido drenado. Tampoco se lavalas manos al final del procedimiento.

\section{Discusión}

El nivel de conocimientos teoricoprácticos que tienen los pacientes del recambio peritoneal permitió observar que la mayoría estuvieron en las categorías buenos y excelentes, sin embargo es preocupante para las investigadoras los que se ubicaron en el rango regular y aquellos que no generaron respuesta alguna frente al reentrenamiento. Este trabajo coincide con la afirmación presentada en el trabajo de Bermúdez y Forigua titulado "Programa de reentrenamiento dirigido al paciente en diálisis peritoneal" realizado en la Clínica San Pedro Claver de Bogotá en el 2002 donde se rescata el impacto positivo del programa, a pesar de que no mostró diferencias significativas en el nivel de conocimientos y prácticas después del reentrenamiento.

-Con esta investigación se corrobora la afirmación de Darlys María Navarro Cabarcas en el trabajo sobre la incidencia y mortalidad por peritonitis bacteriana en los pacientes del programa de diálisis peritoneal del Hospital de San José en el que planteaba que los enfermos tienen conocimientos teóricos y prácticos buenos sobre el recambio peritoneal, pero que al realizar los recambios no los aplican
-Se observó que el paciente cumple a cabalidad los pasos del recambio peritoneal que tienen mayor riesgo de peritonitis. Daugirdas en el manual de diálisis afirma que la vía de entrada de esta en la mayoría de los casos es la intraluminal, área en donde los pacientes demostraron buenos conocimientos prácticos. Es llamativo que la incidencia de peritonitis sigue siendo alta.

\section{Conclusiones}

- Al aplicar la primera encuesta y lista de comprobación se observó que el 55\% de los pacientes tenían buenos conocimientos teoricoprácticos del recambio peritoneal, sólo un 3\% presentó deficiencia en la parte teórica y $26 \%$ mostró práctica regular.

-En el desarrollo del programa de reentrenamiento hubo colaboración por parte de los pacientes y del personal de la unidad renal; además de la participación de los enfermos se incluyó en el programa al familiar más cercano. La metodología utilizada fue la de educación para adultos

-En cuanto a los resultados de los conocimientos teoricoprácticos después del programa de reentrenamiento se observaron mejores resultados, desaparece el comportamiento deficiente en los conocimientos teóricos y disminuye el rango de regular en la práctica en un $50 \%$. Los porcentajes de excelencia aumentaron en forma significativa en los conocimientos teóricos y en los prácticos.

-En los pasos de la técnica del recambio peritoneal se observó que el $34 \%$ de los pacientes no siguen el protocolo recomendado referente a la posición de la mano cuando se realiza la conexión y desconexión del sistema ultrabag.

-En los conocimientos teóricos y prácticos se encontró que los comportamientos de revisar el estado del equipo a utilizar y de verificar las características del ultrabag (fecha de vencimiento, concentración e integralidad) los pacientes no lo realizaron en la teoría $(31 \%)$ ni en la práctica ( $46 \%)$. 
-En cuanto al nivel de educación se observó que la mayoría de los pacientes han cursado primaria; en los pacientes con nivel superior técnico y universitario se encontró que presentaron conocimientos teoricoprácticos excelentes y buenos antes y después del reentrenamiento.

-Se concluyó que el programa de reentrenamiento influyó positivamente en los conocimientos teoricoprácticos del recambio peritoneal ya que los pacientes modificaron sus comportamientos.

-Por ser una muestra tan pequeña la investigación no es concluyente, lo que no excluye que se tomen medidas correctivas donde se encontraron deficiencias.

\section{Recomendaciones}

- Al observar los resultados del programa de reentrenamiento se recomienda mantener un programa de capacitación permanente para pacientes y familiares.

- En pacientes que presenten peritonitis a repetición se debe hacer seguimiento a través de visitas domiciliarias y reentrenamiento individual.
- Organizar un sistema de estímulos o premios para quienes presenten mayor autocuidado y adaptabilidad al tratamiento.

- Crear grupos de apoyo entre los mismos pacientes tomando como líderes a los mejores, para que sirvan de red de apoyo y de orientación.

- Continuar trabajos de investigación con participación de un equipo interdisciplinario con el fin de tener una visión integral del cuidado del paciente y en especial para indagar sobre otros factores que puedan incidir en la peritonitis y lograr soluciones oportunas y eficientes.

\section{Lecturas recomendadas}

- Bermúdez ML., Forigua LA. Programa de reentrenamiento dirigido al paciente en diálisis peritoneal (tesis). Bogotá: Fundación Universitaria de Ciencias de la Salud; 2002.

- Borrero J, Montero O. Fundamentos de Medicina: Nefrología. 4a ed. Medellín: Corporación para Investigaciones Biológicas; 2003.

- Daurgidas J. Manual de diálisis. Buenos Aires: Editorial M $\mathrm{L} ; 2000$.

- Navarro DM. Incidencia y mortalidad por peritonitis bacteriana en los pacientes del programa de diálisis peritoneal del Hospital de San José (tesis). Bogotá: Fundación Universitaria de Ciencias de la Salud; 2000. 